\title{
NONLINEAR INTERACTION OF POSITIVE AND NEGATIVE ENERGY MODES IN HAMILTONIAN SYSTEMS
}

\author{
J. P. DENIER ${ }^{1}$ AND R. H. J. GRIMSHAW ${ }^{2}$
}

(Received 23 August 1988; revised 19 January 1989)

\begin{abstract}
We consider the nonlinear evolution of a Hamiltonian system as the system passes through a linear resonance (as the system parameters vary). Two cases are considered. In the first case the linearized problem (at resonance) possess a full complement of normal mode solutions. This case is presented in the context of the interaction between modes which may have oppositely signed energy. The second case considered has an additional degeneracy in that the linearized problem (at resonance) has a single normal mode solution.

Both cases are analysed using normal form theory and in both cases the systems governing the transition through resonance are shown to be completely integrable in the classical sense. Possible bifurcations as the resonance is traversed are discussed. Conditions for the existence of algebraic singularities at some finite positive time are also presented.
\end{abstract}

\section{Introduction}

In this paper we are interested in the nonlinear evolution of a conservative dynamical system as the linear normal mode solutions pass through resonance. The resonance occurs when two normal frequencies $\omega_{1,2}$ are such that $\left|\omega_{1}\right| \approx\left|\omega_{2}\right|$ (for some parameter value). The energy, $E_{i}$, of the mode corresponding to the frequency $\omega_{i}$ can (though normalization) be related to the sign of the frequency $\omega_{i}$ at the point of resonance, i.e. $\operatorname{sign}\left(E_{i}\right)=\operatorname{sign}\left(\omega_{i}\right)$.

\footnotetext{
'Present address: Department of Mathematics, Exeter University, North Park Road, Exeter, EX4 4QE, England.

${ }^{2}$ School of Mathematics, University of New South Wales, P. O. Box 1, Kensington, NSW 2033, Australia.

(C) Copyright Australian Mathematical Society 1990, Serial-fee code 0334-2700/90
} 
The physical significance of a mode of negative energy is seen when the energy $E$ corresponds to the Hamiltonian function for the linearized equations of motion. A mode then has negative energy when the excitation of this mode lowers the energy level of the system relative to some basic state. For example, in shear flows, for which a mode is a wave on an interface separating two fluids of different densities, exciting a wave of negative energy lowers the total energy of the system relative to the previously unperturbed flow, see Cairns [2], Craik and Adam [3].

The transition through a resonance of the linear normal mode solutions can be classified into two distinct classes depending on the signs of the energy of the two interacting modes (see Arnold [1], App. 6). The case when both normal modes have the same signed energy (i.e. both positive or both negative energy modes) will not be considered here. However, the case when the two interacting modes have oppositely signed energies is of particular interest (this case arises in many physical situations). We will restrict our attention to this case. As will be shown this form of interaction between the linear normal modes gives rise to a single complex mode (which in the linear theory allows for unlimited growth in both modes during the interaction). The production of this complex mode poses the question of how the solutions to the fully nonlinear problem behave as the point of resonance is traversed.

The resonant interaction of positive and negative energy modes in conservative dynamical systems has received considerable attention in the last decade. The linear theory has been considered by Grimshaw, [6], (in the case when the system parameters are slowly-varying functions of the time) who shows that the action in both modes grows during resonance. Applications to resonant interactions (resonant triads $-\omega_{1}+\omega_{2} \pm \omega_{3} \approx 0$ ) in fluids have been considered by Craik and Adams, [3], and Cairns, [2], who show that the presence of both positive and negative energy modes in a resonant traid results in the production of an explosive instability. The general problem of the nonlinear interaction of positive and negative energy modes in resonant traids occurring in Hamiltonian systems has been considered by Grimshaw [18], (again the parameters are slowly varying). However, the case of the triad resonance is inherently nonlinear whereas it is the case of the 2-mode resonance which occurs in linear system. The linear instability caused through the resonant interaction of a positive energy mode with a negative energy mode is characteristic of the Kelvin-Helmholtz instability found in parallel shear flows; see Cairns [2], who considers the problem for both a two-layer and a three-layer shear flow.

The main bodies of work on resonant interactions in Hamiltonian systems is to be found in the literature of plasma physics and celestial mechanics. The celestial mechanics literature provides a wealth of work on this subject, 
most of which is concerned with the restricted three body problem which exhibits such behaviours at the Routhian points (the term Hamiltonian Hopf bifurcation has been coined for problems of the type that we wish to consider; see van der Meer [15], and references therein). In plasma physics instabilities which arise as a result of the interaction of positive- and negative energy modes are known as reactive instabilities, see Davidson, [4], and the references contained therein.

In this paper we consider the nonlinear response of a Hamiltonian system in which a (linear) positive energy mode interacts with a negative energy mode through a resonance. The main question to be considered is the response of the system as the resonance is traversed as some system parameter(s) are varied. We employ the method of normal mode theory and make use of normal forms to reduce the Hamiltonian system to a simpler system (which is still Hamiltonian) which can be studied to yield some of the details of the dynamics of the problem. Two cases are considered and are characterised by the number of normal modes solutions which the linear system admits at the point of resonance.

\section{Semi-simple resonance}

(a) Formulation. In this section we consider a system of 2-degrees-of freedom governed by Hamilton's canonical equations

$$
d \mathbf{q} / d t=\partial H(\mathbf{q}, \mathbf{p}) / \partial \mathbf{p}, \quad-d \mathbf{p} / d t=\partial H(\mathbf{q}, \mathbf{p}) / \partial \mathbf{q}
$$

where $H(\mathbf{q}, \mathbf{p} ; \mu \nu)$ is the Hamiltonian function; $\mathbf{q}=\left(q_{1}, q_{2}\right), \mathbf{p}=\left(p_{1}, p_{1}\right)$ are the generalized coordinates and momenta, respectively, $\mu$ and $\nu$ are system parameters. To discuss the transition of the system (2.1) through a linear resonance, it is convenient to introduce two parameters; these parameters will later be related to each other leaving a single parameter to describe the transition through the resonance. The Hamiltonian is assumed to be analytic in all arguments. We suppose that $\mathbf{p}=\mathbf{q}=0$ is an equilibrium solution of (2.1) for all $\mu$ and $\nu$. The stability of this solution is determined by the linearization of (2.1) about the origin:

$$
d \mathbf{q} / d t=B^{T} \mathbf{q}+C \mathbf{p}, \quad-d \mathbf{p} / d t=B \mathbf{p}+A \mathbf{q}
$$

where

$$
A=\left(\frac{\partial^{2} H}{\partial q_{i} \partial q_{j}}\right)_{0}, \quad B=\left(\frac{\partial^{2} H}{\partial q_{i} \partial p_{j}}\right)_{0}, \quad C=\left(\frac{\partial^{2} H}{\partial p_{i} \partial p_{j}}\right)_{0} .
$$

The subscription 0 indicates that the function is evaluated at the origin in (q, p) space; $T$ indicates the transpose. The system (2.2) has normal mode 
solutions consisting of the triplet $[\omega ; \xi, \eta]$, where

$$
(\mathbf{q}, \mathbf{p})=(\xi, \eta) \exp (i \omega t)
$$

and

$$
i \omega \xi=B^{T} \xi+C \eta ; \quad-i \omega \eta=B \eta+A \xi .
$$

From (2.4) we see that $[-\bar{\omega} ; \bar{\xi}, \bar{\eta}]$ is also a solution. Also, not that the adjoint system to $(2.4)$ is just $(2.4)$ with $\omega$ replaced by $\bar{\omega}$. Thus $[\bar{\omega} ; \hat{\xi}, \hat{\eta}]$ and $[-\omega ; \overline{\bar{\xi}}, \hat{\eta}]$ are solutions of $(2.4)$ (here $\bar{\xi}, \hat{\eta}$ are the adjoint normal modes and are solutions of (2.4) with $\omega$ replaced by $\bar{\omega})$. From (2.4) we can show that

$$
\begin{gathered}
-i(\omega+\bar{\omega})\left(\bar{\xi}^{T} \eta-\bar{\eta}^{T} \xi\right)=2\left(\bar{\xi}^{T} A \xi+\bar{\eta}^{T} B^{T} \xi+\bar{\xi}^{T} B \eta+\bar{\eta}^{T} C \eta\right), \\
-i(\omega-\bar{\omega})\left(\bar{\xi}^{T} \eta-\bar{\eta}^{T} \xi\right)=0 .
\end{gathered}
$$

Similar relations hold for the adjoint normal modes. We note that the right hand side of the first equation is real-valued and can be considered as the averaged Hamiltonian for that mode. Following Grimshaw, [6], we can show that the normal modes can be put into four categories (positive-energy modes, negative-energy modes, complex modes and imaginary modes).

(i) Positive -energy modes: In this case the averaged Hamiltonian is positive-definite. From (2.5b) it follows that the normal frequencies are real. The normal modes are normalized according to

$$
\bar{\xi}^{T} \eta-\bar{\eta}^{T} \xi=i \text {. }
$$

It follows from (2.5a) that the normal frequency $\omega$ is positive.

(ii) Negative-energy modes: In this case the averaged Hamiltonian is negative-definite. The normalization is as in (i), however from (2.5a) we see that the normal frequency $\omega$ is negative.

(iii) Complex modes In this case the averaged Hamiltonian is indefinite, and the right-hand side of (2.5a) vanishes. From (2.4) and (2.5a,b) it follows that

$$
\bar{\xi}^{T} \eta-\bar{\eta}^{T} \xi=\widehat{\xi}^{T} \eta-\widehat{\eta}^{T} \xi=\overline{\vec{\xi}}^{T} \hat{\eta}=\overline{\bar{\eta}}^{T} \hat{\xi}=0,
$$

and the normal modes occur as a quartet, $[\omega ; \xi, \eta],[-\bar{\omega} ; \bar{\xi}, \bar{\eta}],[\bar{\omega} ; \widehat{\xi}, \hat{\eta}]$, $[-\omega ; \overline{\bar{\xi}}, \overline{\hat{\eta}}]$. The normal frequency is complex with nonzero real and imaginary components and so each member of the quartet is distinct. The normal modes are normalized by

$$
\overline{\bar{\xi}}^{T} \eta-\overline{\bar{\eta}}^{T} \xi=i \text {. }
$$

(iv) Imaginary modes In this case the right hand side of (2.5a) vanishes, the normal frequency $\omega$ now being pure imaginary. The normal modes occur in pairs $[\omega ; \xi, \eta]$ and $[\bar{\omega} ; \widehat{\xi}, \hat{\eta}]$. The normal modes are normalized by

$$
\widehat{\eta}^{T} \eta-\widehat{\eta}^{T} \xi=1
$$

and since $\omega=-\bar{\omega}$ we can take $\xi, \eta, \widehat{\xi}, \widehat{\eta}$ to be real valued. 
(b) Linear theory. We assume that at $\mu=\nu=0$ the system (2.3) has normal mode solutions consisting of the pair

$$
\left[\lambda ; \mathbf{a}_{1}, \mathbf{b}_{1}\right],\left[-\lambda ; \overline{\mathbf{a}}_{1}, \overline{\mathbf{b}}_{1}\right]
$$

and the pair

$$
\left[\lambda ; \overline{\mathbf{a}}_{2}, \overline{\mathbf{b}}_{2}\right],\left[-\lambda ; \mathbf{a}_{2}, \mathbf{b}_{2}\right] .
$$

Then at $\mu=\nu=0$ the normal frequencies satisfy

$$
\omega_{1}+\omega_{2}=0 \text {. }
$$

The system is said to be in 1: -1 resonance at $\mu=\nu=0$. We can consider $\lambda(>0)$ as a normal frequency of multiplicity two whose associated normal mode is

$$
\xi_{0}=\alpha_{1} \mathbf{a}_{1}+\alpha_{2} \overline{\mathbf{a}}_{2}, \quad \eta_{0}=\alpha_{1} \mathbf{b}_{1}+\alpha_{2} j \overline{\mathbf{b}}_{2}
$$

where the $\alpha_{i}$ 's are, as yet, undetermined functions of some parameter. Note that this case has been termed the semi-simple Hamiltonian Hopf bifucation since (2.4) possesses a full complement of normal mode solutions (the nonsemi-simple case occurs when (2.4) has an additional degeneracy in that for the normal frequency $\lambda$ there is a single normal mode solution). Here we have assumed that the $a_{i}$ and $b_{i}$ are independent.

Due to the multiplicity of the normal frequency $\lambda$ we can impose the orthogonality conditions

$$
\begin{gathered}
\overline{\mathbf{a}}_{j}^{T} \mathbf{b}_{k}-\overline{\mathbf{b}}_{j}^{T} \mathbf{a}_{k}=i \delta_{j k}, \\
\mathbf{a}_{j}^{T} \mathbf{b}_{k}-\mathbf{b}_{j}^{T} \mathbf{a}_{k}=0 .
\end{gathered}
$$

To obtain the higher order corrections to the normal fequencies we assume that $\mu$ and $\nu$ are both small parameters and relate $\nu$ to $\mu$ by

$$
\nu=\mu \varepsilon \text {. }
$$

We now expand all the relevant quantities in (2.4) in powers of $\mu$

$$
\begin{gathered}
\omega_{1},-\omega_{2}=\lambda+\mu \delta \omega+O\left(\mu^{2}\right), \\
\xi(\eta)=\xi_{0}\left(\eta_{0}\right)+\mu \delta \xi(\delta \eta)+O\left(\mu^{2}\right)
\end{gathered}
$$

and

$$
A=A_{0}+\mu \delta A+O\left(\mu^{2}\right)
$$

where

$$
A_{0}=A(0,0), \quad \delta A=\partial A(0,0) / \partial \mu+\varepsilon \partial A(0,0) / \partial \nu
$$

with similar expressions for $B$ and $C$. Substitution of these expansions into (2.4) yields at $O(\mu)$

$$
\delta \omega E_{j k} \alpha_{k}=\chi_{j k} \alpha_{k}, \quad(j=1,2)
$$


where we have made use of the orthogonality conditions $(2.9 \mathrm{a}, \mathrm{b})$; the $\alpha_{k}$, $(k=1,2)$, are the coefficients in the leading order normal mode vector $\left(\xi_{0}, \eta_{0}\right)$ (see $(2.8))$. Here

$$
\begin{aligned}
& \chi_{11}=\overline{\mathbf{a}}_{1}^{T} \delta A \mathbf{a}_{1}+\overline{\mathbf{b}}_{1}^{T} \delta B^{T} \mathbf{a}_{1}+\overline{\mathbf{a}}_{1}^{T} \delta B \mathbf{b}_{1}+\overline{\mathbf{b}}_{1}^{T} \delta C \mathbf{b}_{1}=\bar{\chi}_{11}, \\
& \chi_{12}=\mathbf{a}_{2}^{T} \delta A \mathbf{a}_{1}+\mathbf{a}_{2}^{T} \delta B^{T} \mathbf{a}_{1}+\mathbf{a}_{2}^{T} \delta B \mathbf{b}_{1}+\mathbf{b}_{2}^{T} \delta C \mathbf{b}_{1}=\bar{\chi}_{21}, \\
& \chi_{22}=\mathbf{a}_{2}^{T} \delta A \overline{\mathbf{a}}_{2}+\mathbf{b}_{2}^{T} \delta B^{T} \overline{\mathbf{a}}_{2}+\mathbf{a}_{2}^{T} \delta B \overline{\mathbf{b}}_{2}+\mathbf{b}_{2}^{T} \delta C \overline{\mathbf{b}}_{2}=\bar{\chi}_{22}
\end{aligned}
$$

and

$$
\left(E_{i j}\right)=\left(\begin{array}{cc}
1 & 0 \\
0 & -1
\end{array}\right)
$$

From (2.10) the $O(\mu)$ "normal frequencies" are seen to be given by

$$
2 \delta \omega^{(r)}=\chi_{11}-\chi_{22} \pm\left[\left(\chi_{11}+\chi_{22}\right)^{2}-4\left|\chi_{12}\right|^{2}\right]^{1 / 2}, \quad r=1,2 .
$$

The corresponding "eigenvectors" are denoted by $\alpha_{k}^{(r)}$. Since the leading order normal modes $\left(\mathbf{a}_{1}, \mathbf{b}_{1}\right),\left(\overline{\mathbf{a}}_{2}, \overline{\mathbf{b}}_{2}\right)$ are independent of both $\mu$ and $\varepsilon$ we may write

$$
\chi_{i j}=P_{i j}+\varepsilon S_{i j}, \quad(i, j=1,2)
$$

where both $P_{i j}$ and $S_{i j}$ are independent of $\varepsilon$. Following Grimshaw, [6], it can be shown that the freedom of choice in choosing the leading order normal modes $\left(\mathbf{a}_{i}, \mathbf{b}_{i}\right)$ allows us to subject the $\alpha_{k}$ 's to an arbitrary unitary transformation which leaves the eigenfrequencies $\delta \omega$ invariant. We choose this transformation to diagonalize $\left(S_{i j}\right)$ and put

$$
\left(S_{i j}\right)=\left(\begin{array}{cc}
S_{1} & 0 \\
0 & -S_{2}
\end{array}\right)
$$

where, without loss of generality, we may assume that $S_{1}>S_{2}$. Also by suitably redefining the parameter $\varepsilon$ we may assume that $P_{11}=P_{22}=0$. Making use of these manipulations we find that $O(\mu)$ corrections to the normal frequencies may now be written as

$$
2 \delta \omega_{1,2}=\varepsilon\left(S_{1}+S_{2}\right) \pm\left[\varepsilon^{2}\left(S_{1}-S_{2}\right)^{2}-4\left|P_{12}\right|^{2}\right]^{1 / 2}
$$

with the corresponding "eigenvectors" given by (2.10). Note that at $\varepsilon=\varepsilon_{0}$ the system (2.10) has a double zero 'eigenvalue' and a single 'eigenvector' $\left(\alpha_{k}\right)$.

From (2.11) we see that $\delta \omega$ is real valued when [] $>0$, while for [] $<0 \delta \omega$ is complex with nonzero real an imaginary parts. It follows from the above analysis that

$$
\omega_{1}=\lambda+\mu \delta \omega_{1}+O\left(\mu^{2}\right), \quad \omega_{2}=-\lambda-\mu \delta \omega_{2}+O\left(\mu^{2}\right)
$$

so that for $\varepsilon= \pm \varepsilon_{0}$ we have an exact resonance given by (2.7) (see Figure 1). Here $\varepsilon_{0}$ is given by

$$
\varepsilon_{0}^{2}\left(S_{1}-S_{2}\right)^{2}-4\left|P_{12}\right|^{2}=0 .
$$




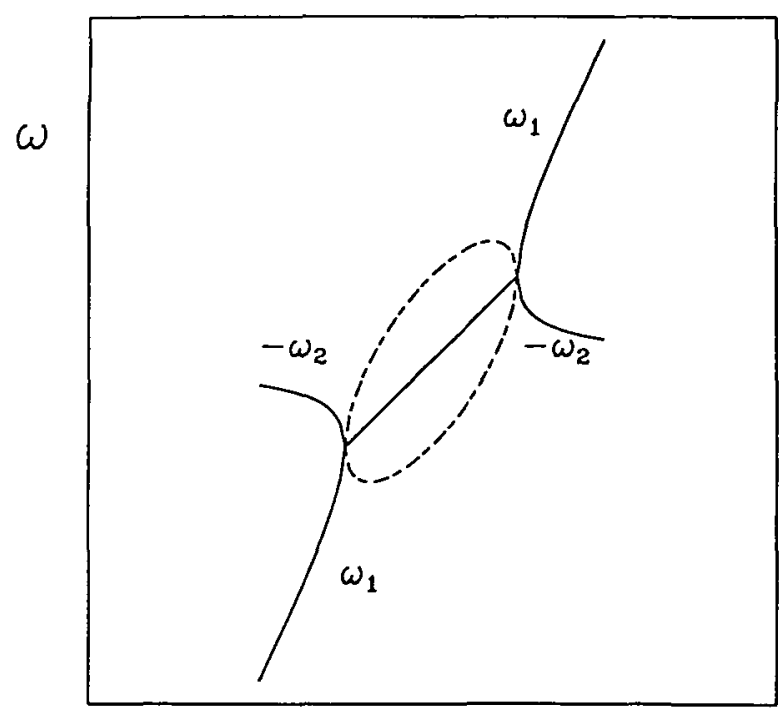

$\varepsilon$

Figure 1. A plot of $\omega_{1,2}$ as a function of $\varepsilon$; dashed curve shows $\operatorname{Im}(\omega)$.

Thus, the consequence of the interaction of a positive and a negative energy mode due to the resonance (2.7) is the 'production' of a complex mode (which exists in the parameter range $\left.|\varepsilon|<\varepsilon_{0}\right)$. At this stage linear theory predicts the exchange of energy between what were the positive and negative energy modes due to the production of the complex mode. We also note that it can be shown that the action is conserved in the parameter region in which the complex mode is present; however this conservation of action allows for exponential growth in both modes when the parameter lies in the region in which the complex mode is present (see Grimshaw, [6]).

We emphasize that these conclusions are based solely upon linear theory; to fully analyse the behavior of the system due to the production of the complex mode we must incorporate the nonlinear terms into the analysis.

(c) Nonlinear theory. Consider the nonlinear problem (2.1). For simplicity of notation we write

$$
H=\sum_{2}^{n} H_{k}(\mathbf{q}, \mathbf{p} ; \mu, \varepsilon)
$$

where $H_{k}(k \geq 2)$, is homogeneous of degree $k$ in $q_{i}, p_{i}$. Each $H_{k}$ is assumed to be analytic in all arguments. To analyse the nonlinear problem we first reduce the system (2.1) with Hamiltonian (2.13) to its normal form. This involves a succession of contact transformations which convert each $H_{k}$ into 
its normal form. We shall use the following procedure for transforming the quadratic Hamiltonian $\mathrm{H}_{2}$ to its normal form. Let

$$
\mathbf{q}=Q_{i} \xi_{i}+\bar{Q}_{i} \bar{\xi}_{i}, \quad \mathbf{q}=Q_{i} \eta_{i}+\bar{Q}_{i} \bar{\eta}_{i}
$$

where the $\left(\xi_{i}, \eta_{i}\right)$ are the normal mode solutions of (2.3) corresponding to the normal frequencies $\omega_{i}$. However this transformation has an inherent singularity which arises through the form of the leading order normal mode $\left(\xi_{0}, \eta_{0}\right)$ given by $(2 . \overline{8})$. This singularity arises due to the presence of the $\alpha_{k}$ 's in (2.8); the $\alpha_{k}$ 's induce a singularity in the transformation at $|\varepsilon|=\varepsilon_{0}$ (since at $|\varepsilon|=\varepsilon_{0}$ there is a single 'eigenvector' $\left(\alpha_{k}\right)$ of the system (2.10). This difficulty can be overcome by instead defining the transformation according to

$$
\mathbf{q}=Q_{i} \mathbf{a}_{i}+\bar{Q}_{i} \overline{\mathbf{a}}_{i}, \quad \mathbf{p}=Q_{i} \mathbf{b}_{i}+\bar{Q}_{i} \overline{\mathbf{b}}_{i}
$$

where the $a_{i}$ 's and $b_{i}$ 's are solutions of (2.4) with $\omega=\lambda$ and satisfy the orthogonality conditions $(2.9 \mathrm{a}, \mathrm{b})$. With such a transformation the system (2.1) becomes

$$
\begin{aligned}
& d Q_{1} / d t=i\left(\lambda+\Lambda_{11}\right) Q_{1}+i \Lambda_{12} \bar{Q}_{2}+i \sum_{3}^{n} \partial H_{3} / \partial \bar{Q}_{1} \\
& d Q_{2} / d t=-i\left(\lambda-\Lambda_{22}\right) Q_{2}+i \Lambda_{12} \bar{Q}_{1}+i \sum_{3}^{n} \partial H_{3} / \partial \bar{Q}_{2}
\end{aligned}
$$

together with the corresponding complex conjugate equations; here $\Lambda_{i j}=$ $\mu \chi_{i j}$ and only the leading order linear terms have been retained. We have made use of the orthogonality conditions $(2.9 \mathrm{a}, \mathrm{b})$ and have assumed that the $a_{i}$ and $b_{i}$ are chosen so as to produce (2.11). If in (2.14a) and (2.14b) we define

$$
P_{i}=-i \bar{Q}_{i}, \quad(1=1,2)
$$

then the transformation from $(\mathbf{q}, \mathbf{p})$ to $(Q, P)$ is a contact transformation (i.e. preserves the Hamiltonian structure of the system). The Hamiltonian is given by

$$
H=\lambda\left(\left|Q_{1}\right|^{2}-\left|Q_{2}\right|^{2}\right)+\Lambda_{11}\left|Q_{1}\right|^{2}+\Lambda_{12} \bar{Q}_{1} \bar{Q}_{2}+\bar{\Lambda}_{12} Q_{1} Q_{2}+\Lambda_{22}\left|Q_{2}\right|^{2}+\sum_{3}^{n} H_{k} .
$$

To transform the higher order terms of the Hamiltonian to their normal form we make use of the method of Lie transforms (see Appendix A). This removes all non-resonant terms from the Hamiltonian function. The Hamiltonian then has the normal form

$$
\begin{aligned}
H= & \lambda\left(\left|u_{1}\right|^{2}-\left|u_{2}\right|^{2}\right)+\Lambda_{11}\left|u_{1}\right|^{2}+\Lambda_{12} \bar{u}_{1} \bar{u}_{2} \\
& +\bar{\Lambda}_{12} u_{1} u_{2}+\Lambda_{22}\left|u_{2}\right|^{2}+\sum_{j k m n}^{b} u_{1}^{j} u_{2}^{k} \bar{u}_{1}^{m} \bar{u}_{2}^{n},
\end{aligned}
$$

the sum being over all indices $j, k, m, n$ such that $j+n=k+m=2$. However 
the Hamiltonian (2.15) must be real, since our original Hamiltonian was real. Thus the nonlinear coefficients must satisfy

$$
b_{j k m n}=\bar{b}_{m n j k},
$$

for all possible indices $j, k, m, n$. Hamilton's canonical equations for the Hamiltonian (2.15) are given by

$$
\begin{aligned}
& d u_{1} / d t=i\left(\lambda+\Lambda_{11}\right) u_{1}+i \Lambda_{12} \bar{u}_{2}+i \sum m b_{j k m n} u_{1}^{j} u_{2}^{k} \bar{u}_{1}^{m-1} \bar{u}_{2}^{n}, \\
& d \bar{u}_{2} / d t=i\left(\lambda-\Lambda_{22}\right) \bar{u}_{2}-i \bar{\Lambda}_{12} u_{1}-i \sum k b_{j k m n} u_{1}^{j} u_{2}^{k-1} \bar{u}_{1}^{m} \bar{u}_{2}^{n}
\end{aligned}
$$

From the linear theory we know that the "bifurcation problem" is governed by the term

$$
\sigma=\left(\Lambda_{11}+\Lambda_{22}\right)-4\left|\Lambda_{12}\right|^{2} .
$$

To facilitate the analysis of the system $(2.16 \mathrm{a}, \mathrm{b})$ as $\sigma$ changes sign we write

$$
\begin{aligned}
& u_{1}=\mu^{1 / 2} d A_{1} / d(\tau)\left[i\left(\lambda+1 / 2\left(\Lambda_{11}+\Lambda_{22}\right)\right) t\right] \\
& \bar{u}_{2}=\mu^{1 / 2} d A_{2} / d(\tau)\left[i\left(\lambda+1 / 2\left(\Lambda_{11}+\Lambda_{22}\right)\right) t\right]
\end{aligned}
$$

where $\tau=\mu t$ and $\Lambda_{i j}=\mu \chi_{i j}$; (this corresponds to removing the oscillatory part of the solution which arises from the real part of the normal frequencies $\omega_{1}$ and $\left.\omega_{2}\right)$. From (2.16a) and (2.16b) we obtain

$$
\begin{aligned}
& d A_{1} / d \tau=(i / 2)\left(\chi_{11}+\chi_{22}\right) A_{1}+i \chi_{12} A_{2}+i \sum m b_{j k m n} A_{1}^{j} \bar{A}_{2}^{k} \bar{A}_{1}^{m-1} A_{2}^{n} \\
& d A_{2} / d \tau=-i \bar{\chi}_{12} A_{1}-(i / 2)\left(\chi_{11}+\chi_{22}\right) A_{2}-i \sum k b_{j k m n} A_{1}^{j} \bar{A}_{2}^{k-1} \bar{A}_{1}^{m} A_{2}^{n}
\end{aligned}
$$

which are Hamilton's canonical equations for the real Hamiltonian

$$
\begin{aligned}
H\left(A_{i} \bar{A}_{i}\right)= & \frac{1}{2}\left(\chi_{11}+\chi_{22}\right)\left(\left|A_{1}\right|^{2}+\left|A_{2}\right|^{2}\right) \\
& +\chi_{12} A_{2} \bar{A}_{1}+\bar{\chi}_{12} A_{1} \bar{A}_{2}+\sum b_{j k m n} A_{1}^{j} A_{2}^{n} \bar{A}_{1}^{m} \bar{A}_{2}^{k},
\end{aligned}
$$

the canonical variables being $\left(A_{1}, A_{2}\right)$ and $\left(-i \bar{A}_{1}, i \bar{A}_{2}\right)$. The system $(2.17 \mathrm{a}, \mathrm{b})$ can now be studied to determine what bifurcations occur as the parameter $\sigma$ changes sign.

Before proceeding there are several points to note from (2.17a) and (2.17b). Firstly, it is easily shown that the system possesses the integral of motion

$$
I=\left|A_{1}\right|^{2}-\left|A_{2}\right|^{2}=\text { constant }
$$

which represents the conservation of action in both modes. Note that this conservation law allows for unlimited growth in the two modes (cf section $2(b))$. Since the two degree of freedom system governed by $(2.17 \mathrm{a}, \mathrm{b})$ is Hamiltonian and possess two integrals of motion, namely the action and 
the "energy" given by the Hamiltonian (2.18), it is completely integrable in the classical sense.

Returning to the original system $(2.16 \mathrm{a}, \mathrm{b})$ we note that according to Liapunov, [12], this system possesses two families of periodic solutions in the parameter range for which the normal frequencies are real. Also, applying the stable manifold theorem, [9], to the system $(2.17 \mathrm{a}, \mathrm{b})$ (or equivalently to $(2.16 \mathrm{a}, \mathrm{b}))$ we find that in the parameter range $\sigma<0$ there can be no periodic solutions in a neighbourhood of the origin in $\left(u_{1}, u_{2}\right)$-space (since in this region of the parameter space the normal frequencies are complex and hence the origin in $\left(u_{1}, u_{2}\right)$-space is a hyperbolic fixed point of the system (2.1)). Thus, any peridic solutions which exist in this parameter range must be of finite amplitude.

(d) Perturbation analysis. First we will restrict our attention to a neighbourhood of the resonance points $\varepsilon= \pm \varepsilon_{0}$. Consider the linearized system corresponding to $(2.17 \mathrm{a}, \mathrm{b})$

$$
\begin{aligned}
& d A_{1} / d \tau=\frac{i}{2} \varepsilon\left(S_{1}-S_{2}\right) A_{1}+i P_{12} A_{2}, \\
& d A_{2} / d \tau=-i \bar{P}_{12} A_{1}-\frac{i}{2} \varepsilon\left(S_{1}-S_{2}\right) A_{2} .
\end{aligned}
$$

(where we have made use of the normalization discussed in the linear theory). From the linear theory we know that at $\varepsilon= \pm \varepsilon_{0}$ this linearized system has a double zero eigenvalue and a single corresponding normal mode vector. Here $\varepsilon_{0}$ is given by (2.12). We will restrict our attention to a neighbourhood in $\varepsilon$-space about $\varepsilon=\varepsilon_{0}$.

Define the matrix

$$
\Lambda_{0}=\left(\begin{array}{cc}
\frac{1}{2} \varepsilon_{0}\left(S_{1}-S_{2}\right) & P_{12} \\
-\bar{P}_{12} & -\frac{1}{2} \varepsilon_{0}\left(S_{1}-S_{2}\right)
\end{array}\right)=\left|P_{12}\right|\left(\begin{array}{cc}
1 & e^{i \varphi} \\
-e^{-i \varphi} & -1
\end{array}\right)
$$

where we have set $P_{12}=\left|P_{12}\right| e^{i \varphi}$ and made use of the definition of $\varepsilon_{0}$. We define the normal and generalized normal mode vectors according to

$$
\Lambda_{0} \xi_{1}=0, \quad \Lambda_{0} \xi_{2}=\xi_{1} \text {. }
$$

For example

$$
\xi_{1}=2\left(1,-e^{-i \varphi}\right)^{T}, \quad \xi_{2}=\left|P_{12}\right|^{-1}\left(1, e^{-i \varphi}\right)^{T} .
$$

We also define adjoint vectors

$$
\Lambda_{0}^{*} \xi_{2}^{*}=0, \quad \Lambda_{0} \xi_{1}^{*}=\xi_{2}^{*}
$$

where $\Lambda_{0}^{*}=\bar{\Lambda}_{0}^{T}$. Comparing $(2.19 \mathrm{a}, \mathrm{b})$ we can choose

$$
\begin{array}{ll}
\xi_{21}^{*}=\xi_{11}, & \xi_{22}^{*}=-\xi_{12}, \\
\xi_{11}^{*}=\xi_{21}, & \xi_{12}^{*}=-\xi_{22},
\end{array}
$$


where $\xi_{j}=\left(\xi_{j 1}, \xi_{j 2}\right)^{T}$, etc. We have the natural orthogonality conditions

$$
\begin{gathered}
\left\langle\xi_{2}, \xi_{2}^{*}\right\rangle=0, \quad\left\langle\xi_{2}, \xi_{1}^{*}\right\rangle=\beta, \\
\left\langle\xi_{1}, \xi_{1}^{*}\right\rangle=\left\langle\xi_{2}, \xi_{2}^{*}\right\rangle=\alpha,
\end{gathered}
$$

where we are still free to choose the real constants $\alpha, \beta$ with a suitable choice of the normal mode vectors $\xi_{1}$ and $\xi_{2}$. Here $\langle\mathbf{v}, w\rangle=v_{k} \bar{w}_{k}$ is the usual Euclidean dot product.

Returning to the fully nonlinear system we make the transformation

$$
\mathrm{A}=\left(A_{1}, A_{2}\right)^{T}=B_{1} \xi_{1}+B_{2} \xi_{2} .
$$

If we choose the variables $Q_{j}=B_{j}$ and $P_{1}=-i \bar{B}_{2}, P_{2}=-i \bar{B}_{1}$ then the transformation from the canonical variables $\left(A_{1}, A_{2}\right),\left(-i \bar{A}_{1}, i \bar{A}_{2}\right)$ to the variables $\left(Q_{j}, P_{j}\right)$ is canonical that $\xi_{1}$ and $\xi_{2}$ (i.e. $\alpha$ and $\beta$ ) are chosen such that

$$
\beta=0, \quad \alpha=1 \text {. }
$$

The transformed Hamiltonian is then given by

$$
\begin{aligned}
\widehat{H}= & \left|B_{2}\right|^{2}+\sigma\left[\alpha_{1}\left|B_{1}\right|^{2}+\alpha_{2} B_{1} \bar{B}_{2}+\bar{\alpha}_{2} \bar{B}_{1} B_{2}+\alpha_{3}\left|B_{2}\right|^{2}\right] \\
& +\sum d_{j k m n} B_{1}^{j} B_{2}^{n} \bar{B}_{1}^{m} \bar{B}_{2}^{k}
\end{aligned}
$$

where

$$
\begin{gathered}
\sigma=\varepsilon-\varepsilon_{0} \\
2 \alpha_{1}=\left(S_{1}-S_{2}\right)\left(\left|\xi_{11}\right|^{2}+\left|\xi_{12}\right|^{2}\right)>0, \\
2 \alpha_{2}=\left(S_{1}-S_{2}\right)\left(\xi_{11} \bar{\xi}_{21}+\xi_{12} \bar{\xi}_{22}\right), \\
2 \alpha_{3}=\left(S_{1}-S_{2}\right)\left(\left|\xi_{21}\right|^{2}+\left|\xi_{22}\right|^{2}\right)>0
\end{gathered}
$$

and the $d_{j k m n}$ are related to the $b_{j k m n}$ of (2.18). In particular

$$
d_{j k m n}=\bar{d}_{m n j k} \text {. }
$$

Hamilton's canonical equations for the Hamiltonian (2.20) are

$$
\begin{gathered}
d B_{1} / d \tau=i B_{2}+i \sigma\left[\alpha_{2} B_{1}+\alpha_{3} B_{2}\right]+i \sum k d_{j k m n} B_{1}^{j} B_{2}^{n} \bar{B}_{1}^{m} \bar{B}_{2}^{k-1} \\
d B_{2} / d \tau=i \sigma\left[\alpha_{1} B_{1}+\bar{\alpha}_{2} B_{2}\right]+i \sum m d_{j k m n} B_{1}^{j} B_{2}^{n} \bar{B}_{1}^{m-1} \bar{B}_{2}^{k} .
\end{gathered}
$$

Since we are interested in a small neighborhood of $\sigma=0$ we rescale

$$
B_{1}=\delta C_{1}, \quad B_{2} C_{1}, \quad B_{2}=\delta^{2} C_{2}, \quad x=\delta \tau, \quad \sigma=\delta^{2} \omega .
$$

To leading order in $\delta$, we obtain from $(2.21 \mathrm{a}, \mathrm{b})$

$$
\begin{gathered}
d C_{1} / d x=i C_{2}, \\
d C_{2} / d x=i \alpha_{1} \omega C_{1}+2 i d_{2020} C_{1}\left|C_{1}\right|^{2}
\end{gathered}
$$


which are Hamilton's canonical equations for the Hamiltonian

$$
G=\left|C_{2}\right|^{2}+\alpha_{1} \omega\left|C_{1}\right|^{2}+d_{2020}\left|C_{1}\right|^{4}=g .
$$

Here $g \in \mathbf{R}$ is the 'total energy' for the reduced system (2.22a,b). Also note that the system $(2.22 \mathrm{a}, \mathrm{b})$ possesses a second integral of motion

$$
2 J=\bar{C}_{2} C_{1}+\bar{C}_{1} C_{2} \text {. }
$$

which is the action integral and represents the conservation of action in both modes. The system $(2.22 \mathrm{a}, \mathrm{b})$ is equivalent to

$$
d^{2} C_{1} / d x^{2}=-\alpha_{1} \omega C_{1}-2 d_{2020} C_{1}\left|C_{1}\right|^{2} .
$$

Equation (2.23) has arisen in the study of Kelvin-Helmholtz instability in a two layer shear flow, see Miles [14], and also in the interaction of a positive energy wave with a negative energy wave in a three layer stratified shear flow where in both cases $\alpha_{1} \omega$ is interpreted as a perturbation about a critical wavenumber, see [5]. We note that the above analysis also holds if we consider a neighborhood of $\varepsilon=-\varepsilon_{0}$.

Before proceeding with an analysis of the solutions to $(2.22 \mathrm{a}, \mathrm{b})$ we consider the steady solutions

$$
C_{2}=0, \quad C_{1}=0
$$

and

$$
C_{2}=0, \quad\left|C_{1}\right|^{2}=-\omega /(2 d),
$$

which correspond to periodic solutions of our original system $(2.16 \mathrm{a}, \mathrm{b})$. Note that as $\omega$ changes sign there is a bifurcation from one equilibrium solution to two equilibrium solutions. In this reduced system this bifurcation is equivalent to a pitchfork bifurcation whereas in our original system governed by the Hamiltonian (2.15) this bifurcation corresponds to a bifurcation to periodic solutions from the trivial equilibrium $(0,0)$. The second of these solutions exists for $d<0, \omega>0$, and $d>0, \omega<0$. However, these solutions can be shown to be stable only for $\omega<0\left(\varepsilon<\varepsilon_{0}\right)$. Note that in the case $\omega<0$ the origin in $\left(C_{1}, C_{2}\right)$-space in unstable. Hence it is only possible for a bifurcation to manifest itself in the second of these cases. So as $\omega$ increases from negative values to positive values the solutions governed by $(2.24 \mathrm{~b})$ tend to zero and no longer exist for $\omega>0$. Thus, the case of bifurcation of periodic solutions of the original system governed by the steady solutions $(2.24 \mathrm{a}, \mathrm{b})$ corresponds to one of the possible behaviours of such systems mentioned by Meyer, [4], in which the family of periodic solutions exist only for $\omega<0$. It would appear that the periodic solutions given by (2.24b) are precisely those predicted by Liapunov's theorem, [12].

Equation (2.23) can be integrated; introducing

$$
C_{1}=R e^{i \theta} ; \quad R, \theta \in \mathbf{R}
$$


we obtain upon equating the real and imaginary components of the resulting equation

$$
\begin{gathered}
d^{2} R / d x^{2}-R(d \theta / d x)^{2}=-g_{1} \omega R-2 d_{2020} R^{3} \\
2 \frac{d \theta}{d x} \frac{d R}{d x}+\frac{d^{2} \theta}{d x^{2}} R=0 .
\end{gathered}
$$

From (2.25b) we obtain

$$
R^{2} \theta_{x}=\text { constant }=J
$$

where $J$ is the action given by (2.22d). Substituting back into (2.25a) yields

$$
d^{2} R / d x^{2}-J^{2} R^{-3}=-\alpha_{1} \omega R-2 d_{2020} R^{3} .
$$

Without loss of generality we will set $\alpha_{1}=1$ and drop the subscripts on the $d_{2020}$. Defining $S=R^{2}$ we obtain from (2.26)

$$
\left(S_{x}\right)^{2}=4\left(g S-\omega S^{2}-d S^{3}-J^{2}\right)
$$

where $g$ is a constant of integration (it is, in fact, the total energy given by (2.22c)). Equation (2.27) can now be integrated in terms of elliptic integrals. Thus

$$
\int\left[g S-\omega S^{2}-d S^{3}-J^{2}\right]^{1 / 2} d S= \pm 2 x+x_{0}
$$

Returning to equation (2.25a) we note that this equation has been derived by Miles, [14], as the equation governing the onset of Kelvin-Helmholtz instability in a two layer stratified fluid. This equation also arises in the study of the interaction of two modes of oppositely signed energy in a three layer stratified shear flow, [5].

Closed trajectories of (2.27) correspond to periodic solutions $R$ of (2.26). The existence of closed trajectories of $(2.27)$ in the $\left(S_{x}, S\right)$ plane depends upon the nature of the roots of the polynomial

$$
P(S)=g S-\omega S^{2}-d S^{3}-J^{2} .
$$


We note that only the positive roots of $P(S)$ correspond to realistic solutions $R$ of (2.26).

Miles, [14], has classified the solutions of (2.27) into the following four categories (where without loss of generality we may assume that $d$ takes only the values \pm 1$)$.

(i) $\omega>0, d=-1 . P(S)$ has three positive ronts. The trajectories in the $\left(S_{\tau}, S\right)$-plane can be both open and closed provided

(ia) $\omega^{2} / 3 \geq g>0$

(ib) $P\left(S_{0}^{-}\right)>0, P\left(S_{0}^{+}\right)<0, S_{0}^{ \pm}=1 / 3\left[\omega \pm\left(\omega^{2}-3 g\right)^{1 / 2}\right]$.

Violation of any of these conditions reduces the number of positive roots of $P(S)$ to one and no closed trajectories exist in the $\left(S_{\tau}, S\right)$-plane (see Figure 2a,b).

(ii) $\omega<0, d=-1, P(S)$ has a single positive root and no closed trajectories exist (see Figure 2c,d).

(iii) $\omega>0, d=1$. In this case $P(S)$ has two positive roots. Closed trajectories exist in the $\left(S_{\tau}, S\right)$-plane provided that the following are satisfied (see Figure 2e,f)

(iiia) $g>0$

(iiib) $P\left(S_{0}\right)>0, S_{0}=1 / 3\left[\left(\omega^{2}+3 g\right)^{1 / 2}-\omega\right]$.
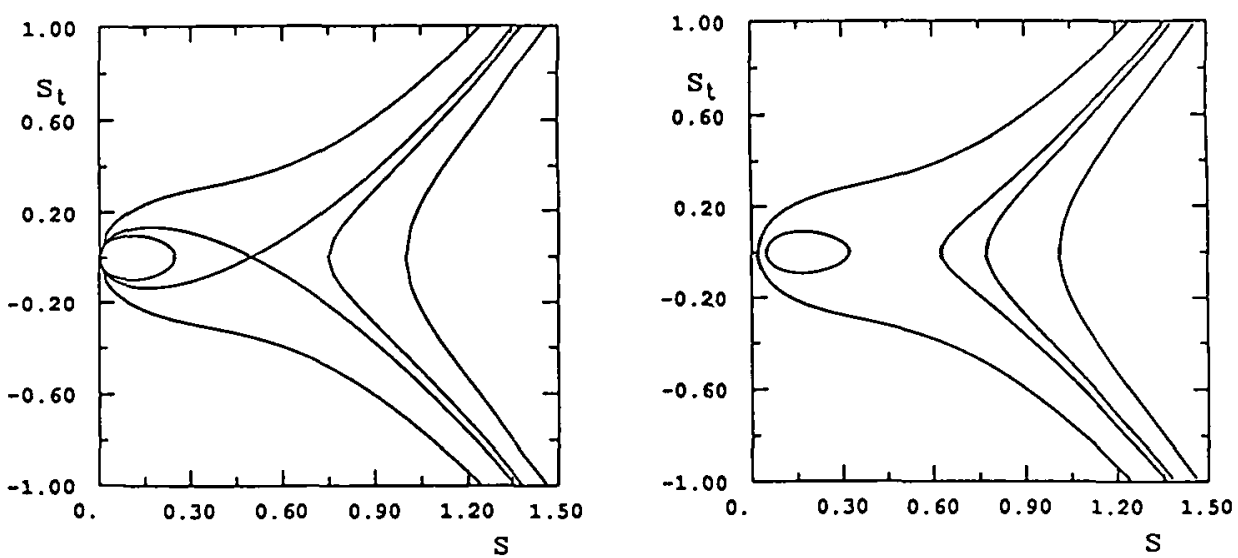

Figure 2a,b. Phase-plane plot of $S_{T}$ versus $S$ for equation $(2.26) ; \omega=1, d=-1$, (a) $J^{2}=0$, (b) $J^{2}=0.01$ and $G=0,0.1875,-.25,0.5$. 

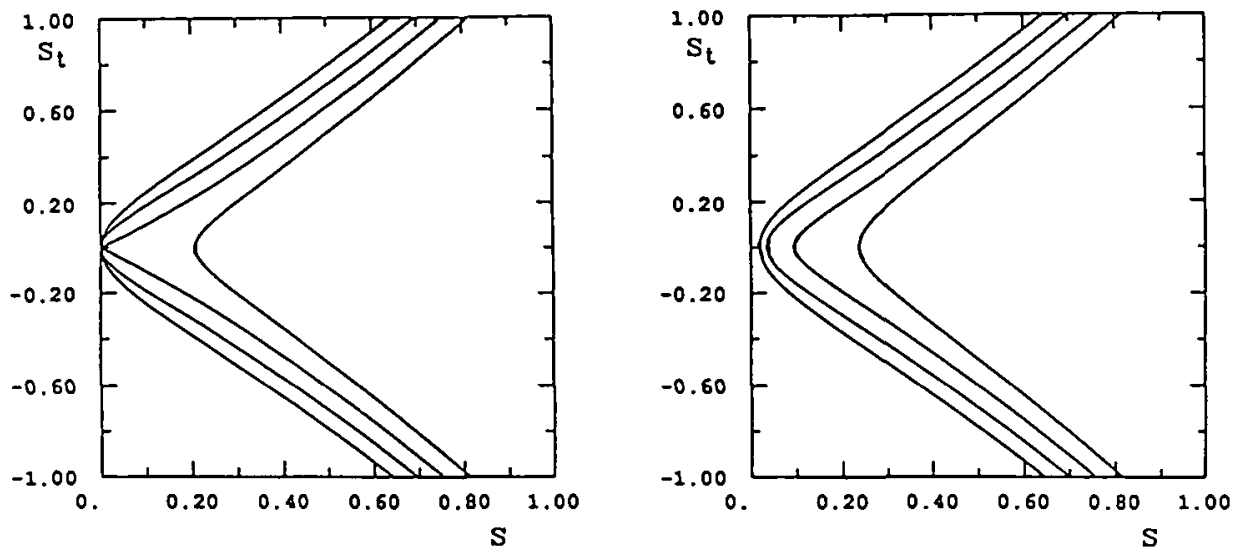

Figure 2c,d. Phase-plane plot of $S_{T}$ versus $S$ for equation (2.26); $\omega=-1, d=-1$, (c) $J^{2}=0$, (d) $J^{2}=0.01$ and $g=-0.25,0,0.25,0.5$.
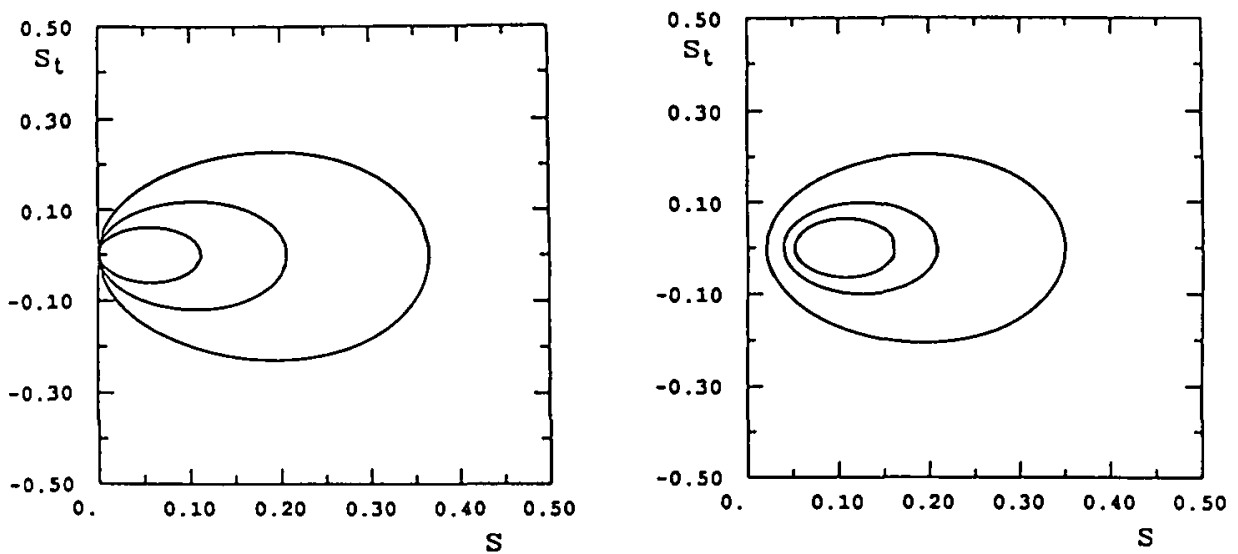

Figure 2e,f. Phase-plane plot of $S_{T}$ versus $S$ for equation $(2.26) ; \omega=1, d=1$. (e) $J^{2}=0, g=0.125,0.25,0,0.5$, (h) $J^{2}=0.001, g=0,0.125,0.25,0.5$.

(iv) $\omega<0, d=1$. In this case $P(S)$ has two positive roots. Closed trajectories exist provided that (see Figure $2 \mathrm{~g}, \mathrm{~h}$ )

(iva) $P\left(S_{0}\right)>0, S_{0}=1 / 3\left[\left(\omega^{2}+3 g\right)^{1 / 2}+|\omega|\right], g \in \mathbf{R}, g \geq-\omega^{2} / 3$.

In cases (ii) and (iv) $S_{0}$ is the positive root of $P_{S}$ (the derivative of $P$ with respect to $S$ ). For these two cases the period of the closed trajectories is given 

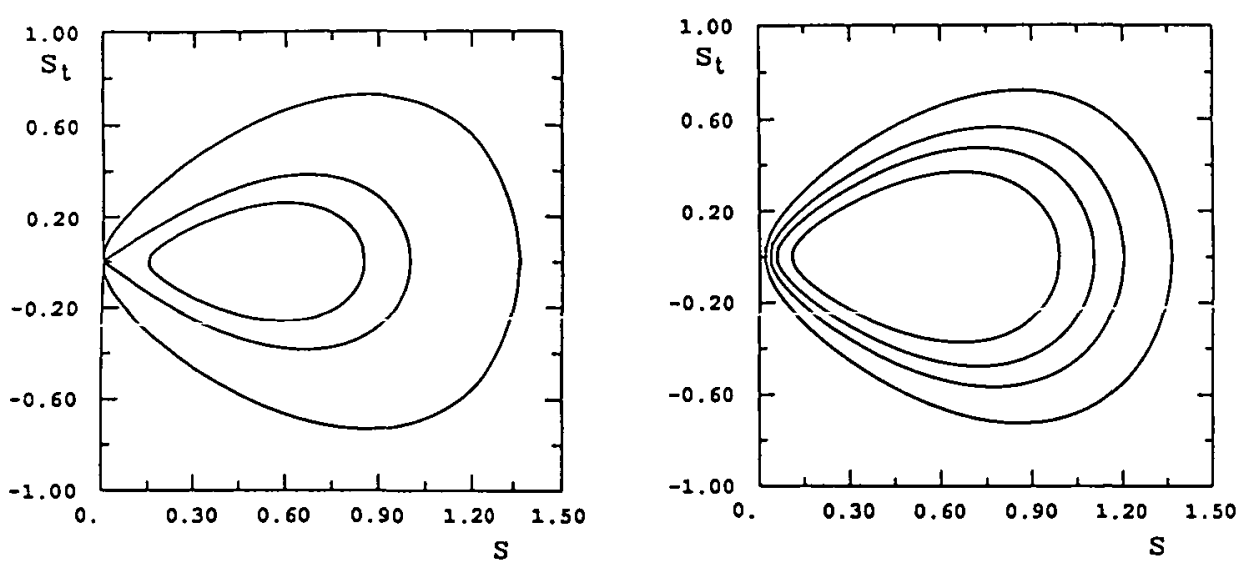

Figure 2g,h. Phase-plane plot of $S_{T}$ versus $S$ for equation (2.26); $\omega=-1, d=1$. (g) $J^{2}=0, g=-0.125,-0.25,0,0.5$, (h) $J^{2}=0.01, g=0,-01.25,0.25,0.5$.

by

$$
\begin{aligned}
T & =\int_{s_{2}}^{s_{1}}\left[\left(s_{1}-s\right)\left(s-s_{2}\right)\left(s-s_{3}\right)\right]^{1 / 2} d s \\
& =2\left(s_{1}-s_{3}\right)^{-1 / 2} K\left[\left(\frac{s_{1}-s_{2}}{s_{1}-s_{2}}\right)^{1 / 2}\right]
\end{aligned}
$$

where $s_{1}>s_{2}>0>s_{3}$ are the roots of $P(S)$.

In cases (i) the period of the closed orbits is given by

$$
\begin{aligned}
T & =\int_{s_{3}}^{s_{2}}\left[\left(s_{1}-s\right)\left(s_{2}-s\right)\left(s-s_{3}\right)\right]^{-1 / 2} d s \\
& =2\left(s_{1}-s_{3}\right)^{-1 / 2} K\left[\left(\frac{s_{2}-s_{3}}{s_{1}-s_{3}}\right)^{1 / 2}\right]
\end{aligned}
$$

where $s_{1}>s_{2}>s_{3}>0$ are the roots of $P(S)$. $K$ is a complete elliptic integral of the first kind. Trajectories in $\left(S_{x}, S\right)$-plane are plotted in Figures 2a-h for various values of the parameters $g, J, \omega, d$.

Considering cases (i)-(iv) we see that there are two possible distinct forms of bifurcation which can occur as the parameter $\omega$ changes sign. The first of these is as described above for the steady solutions. Namely, for $\omega>0$ there exist periodic solutions which tend to zero (disappear) as $\omega$ changes sign. This form of bifurcation is also possible in case (iii) and (iv) provided that the change in sign of the parameter $\omega$ produces a violation of one of the conditions (iib) or (iva). The other form of bifurcation is represented solely by cases (ii) and (iv). This case can be characterised as follows: for $\omega<0$ there exist periodic solutions which persist for $\omega=0$ and for $\omega>0$ they grow 
in amplitude. The fact that these periodic solutions must necessarily grow in amplitude is not evident from the analysis presented above. However, it is possible to infer this property from the stable manifold theorem [9], which implies that there can be no periodic solutions in a neighborhood of the origin for $\omega>0$ (since the linearized normal frequencies are them complex implying linear instability). However, case (iii) ensures that periodic solutions do exist and therefore must be of finite amplitude (which increases with increasing $\omega>0)$.

The analysis about $\varepsilon=-\varepsilon_{0}$ parallels that above. Now, writing, $\varepsilon=-\varepsilon_{0}-\sigma$ we obtain an equation similar to (2.23).

(e) Singular solutions. Let us now return to the system $(2.17 \mathrm{a}, \mathrm{b})$. Due to the presence of the cubic nonlinearities one would expect that solutions of $(2.17 \mathrm{a}, \mathrm{b})$ could posses movable singularities at some finite time $\tau_{0}$. To investigate this possibility we introduce polar coordinates according to

$$
A_{j}=R_{j} \exp \left(i \theta_{j}\right), \quad R_{j}, \theta_{j} \in \mathbf{R}, \quad(j=1,2) .
$$

We write

$$
b_{j k m n}=c_{j k m n}+i d_{j k m n}
$$

and noting that $b_{j k m n}=\bar{b}_{m n j k}$ we obtain, upon equating real and imaginary parts of $(2.17 \mathrm{a}, \mathrm{b})$, the 'amplitude' equations

$$
\begin{aligned}
& d R_{1} / d \tau=-\frac{1}{2} \sin (\varphi-\theta) R_{2}+\left(c_{1021} R_{1}^{2}+c_{0112} R_{2}^{2}\right) R_{2} \sin \theta \\
& -\left(d_{1021} R_{1}^{2}+d_{0112} R_{2}^{2}\right) R_{2} \cos \theta+2\left(c_{2200} \sin 2 \theta+d_{2200} \cos 2 \theta\right) R_{1} R_{2}^{2}, \\
& d R_{2} / d \tau=-\frac{1}{2} \sin (\varphi-\theta) R_{1}+\left(c_{1021} R_{1}^{2}+c_{0112} R_{2}^{2}\right) R_{1} \sin \theta \\
& -\left(d_{1021} R_{1}^{2}+d_{0112} R_{2}^{2}\right) R_{1} \cos \theta \\
& +2\left(c_{2200} \sin 2 \theta+d_{2200} \cos 2 \theta\right) R_{1}^{2} R_{2}
\end{aligned}
$$

and the 'phase' equations

$$
\begin{aligned}
R_{1} d \theta_{1} / d \tau= & (\varepsilon / 2) R_{1}+(1 / 2) \cos (\varphi-\theta) R_{2}+3\left(c_{1021} \cos \theta+d_{1021} \sin \theta\right) R_{1}^{2} R_{2} \\
& +\left(c_{0112} \cos \theta+d_{0112} \sin \theta\right) R_{2}^{3} \\
& +2\left(c_{2200} \cos 2 \theta-d_{2200} \sin 2 \theta\right) R_{2} R_{2}^{2} \\
& +2 c_{2020} R_{1}^{3}+c_{1111} R_{1} R_{2}^{2} \\
R_{2} d \theta_{2} / d \tau= & -(1 / 2) \cos (\varphi-\theta) R_{1}-(\varepsilon / 2) R_{2}-\left(c_{1021} \cos \theta+d_{1021} \sin \theta\right) R_{1}^{3} \\
& -3\left(c_{0112} \cos \theta+d_{0112} \sin \theta\right) R_{2} R_{2}^{2} \\
& -2\left(c_{2200} 2 \theta-d_{2200} \sin 2 \theta\right) R_{1}^{2} R_{2} \\
& -2 c_{0202} R_{2}^{3}-c_{1111} R_{1}^{2} R_{2},
\end{aligned}
$$


where $\theta=\theta_{1}-\theta_{2}$ and we have set (for simplicity) $S_{1}-S_{2}=1, P_{12}=$ $\exp (i \varphi)$. Note that the parameter governing the resonance, $\varepsilon$, appears only in the equation for the phase $\theta_{1}, \theta_{2}$.

A simple dominant balance argument shows that if the amplitude equations $(2.29 \mathrm{a}, \mathrm{b})$ have solutions $\boldsymbol{R}_{j},(j=1,2)$ which develop a singularity at some finite time $\tau_{0}$ then this singularity must be of the form $\left(\tau_{0}-\tau\right)^{-1 / 2}$, $\left(\tau \rightarrow \tau_{0}^{-}\right)$. We then look for solutions of $\left(2.29_{a}-d\right)$ in the fỡil

$$
R_{j}=\left(\tau_{0}-\tau\right)^{-1 / 2} P_{j}, \quad \theta=\theta_{0} \quad\left(\tau \rightarrow \tau_{0}^{-}\right) .
$$

Note that the position of the singularity cannot be predicted i.e. it must be a moveable singularity. From the conservation of action equation we obtain

$$
P_{1}^{2}-P_{2}^{2}=0 \text {. }
$$

With the leading order behaviour given as above we obtain from the amplitude equations $(2.29 \mathrm{a}, \mathrm{b})$ the single equation

$$
\begin{gathered}
2\left[\left(c_{1021}+c_{0112}\right) \sin \theta_{0}-\left(d_{1021}+d_{0112}\right) \cos \theta_{0}\right] P_{1} P_{2} \\
+4\left(c_{2200} \sin 2 \theta_{0}+d_{2200} \cos 2 \theta_{0}\right) P_{1}^{2}-1=0 .
\end{gathered}
$$

From the phase equations $(2.29 c, d)$ we obtain

$$
\begin{aligned}
& {\left[2 c_{2020}+c_{1111}+2\left(c_{2200} \cos 2 \theta_{0}-d_{2200} \sin 2 \theta_{0}\right)\right] P_{1}^{2}} \\
& \quad+\left[\left(3 c_{1021}-c_{0112}\right) \cos \theta_{0}+\left(3 d_{1021}+d_{0112}\right) \sin \theta_{0}\right] P_{1} P_{2}=0(2.30 \mathrm{~b})
\end{aligned}
$$

and

$$
\begin{aligned}
& {\left[2 c_{0202}+c_{1111}+2\left(c_{2200} \cos 2 \theta_{0}-d_{2200} \sin 2 \theta_{0}\right)\right] P_{1}^{2}} \\
& \quad+\left[\left(c_{1021}+3 c_{0112}\right) \cos \theta_{0}+\left(d_{1021}+3 d_{0112}\right) \sin \theta_{0}\right] P_{1} P_{2}=0 .
\end{aligned}
$$

we note that the equations $(2.30 \mathrm{a}-\mathrm{c})$ do not depend upon the parameter, $\varepsilon$, governing the linearized stability.

Equations $(2.30 \mathrm{a}-\mathrm{c})$ are three equations for two unknowns, namely $P_{1}$ and $\theta_{0}\left(P_{2}\right.$ is determined form $\left.P_{2}^{2}=P_{1}^{2}\right)$. We can then consider equation $(2.30 \mathrm{a}, \mathrm{b})$ as equations for the amplitude $P_{1}$, and the phase $\theta_{0}$, respectively. The third equation is then a compatability equation for the nonlinear coefficients, $\left(c_{j k m n}, d_{j k m n}\right)$. Thus, given any particular set of nonlinear coefficients we can predict from $(2.30 \mathrm{a}-\mathrm{c})$ whether the system $(2.17 \mathrm{a}, \mathrm{b})$ can possess singular solutions.

In Figures 3 And 4 we present numerical solutions of the system (2.29a-d). In Figure 3 we have $d_{1021}=1$ with all other nonlinear coefficients set equal to zero. In this case we predict from $(2.30 \mathrm{~b}, \mathrm{c})$ that $\sin \theta_{0}=0$. From (2.30a) we then predict that $\cos \theta_{0}=1$ (in order for the singularity to occur at some 

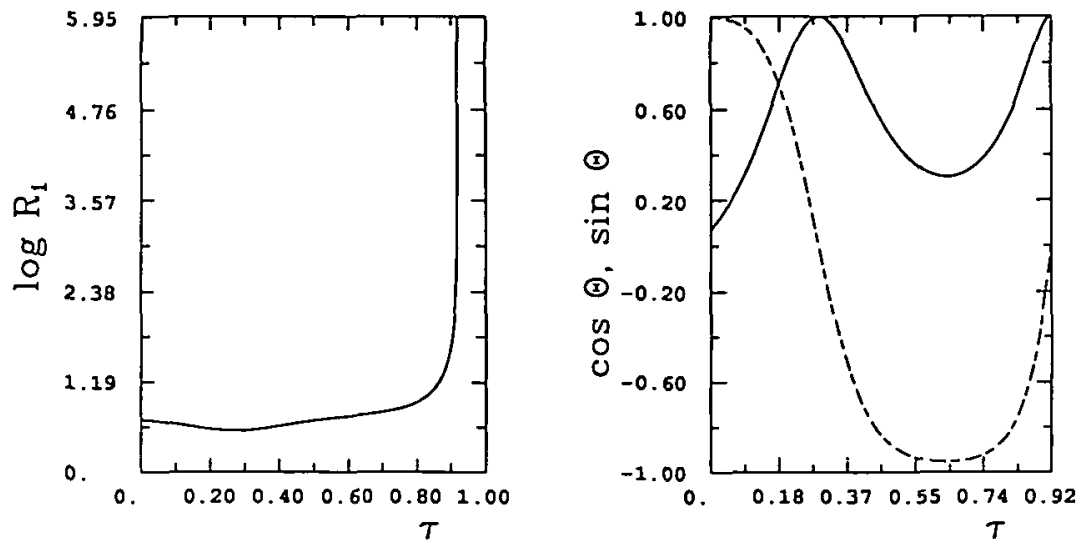

FIGURE 3. Numerical solutions of $(2.29 \mathrm{a}-\mathrm{d})$. Nonlinear coefficients $d_{1021}=1$, with all others equal to zero, $\varepsilon=1, \varphi=\pi$. Plot of $\log r_{1}, \cos \theta$ (solid curve) and $\sin \theta$ (dashed curve) versus $\tau$.
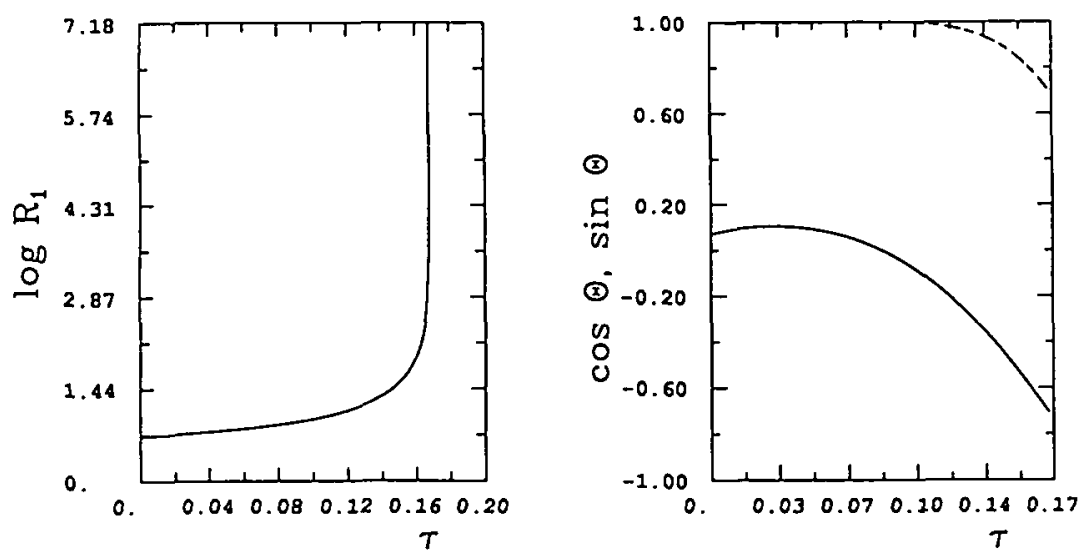

FiguRE 4. Numerical solutions of $(2.29 \mathrm{a}-\mathrm{d})$. Nonlinear coefficients $d_{1021}=c_{1021}=1$ with all others equal to zero, $\varepsilon=0.5, \varphi=\pi$. Plot of $\log R_{1}, \cos \theta$ (solid curve) and $\sin \theta$ (dashed curve) versus $\tau$.

positive time $\tau_{0}>0$ ). The plots of $\log R_{1}, \cos \theta$ and $\sin \theta$ versus $\tau$ in Figure 3 confirm this prediction.

In Figure 4 we have $d_{1021}=c_{1021}=1$ with all other nonlinear coefficients set equal to zero. From $(2.30 \mathrm{~b}, \mathrm{c})$ we predict that $\cos \theta_{0}=-\sin \theta_{0}$. From (2.30a) we then see that $\sin \theta_{0}>0$. The plot of $\log R_{1}, \cos \theta$ and $\sin \theta$ versus $\tau$ in Figure 4 confirm this prediction. 


\section{Nonsemi-simple resonance}

In this section we consider the case of bifurcation from resonance in the nonsemi-simple case. This case has been considered by Van der Meer, [15, 16], whose interest in the problem was motivated by the fact that this type of bifurcation problem occurs in the restricted problem of three bodies at one of the Routhian points. Our interest in this problem is motivated by the occurrence of problems of this type in the interaction of positive and negative energy modes in three layer stratified fluid flows in which the additional degeneracy manifest itself through the particular form of the dispersion relation one encounters in such problems. Van der Meer, [15], approached this problem through the use of real normal forms by making use of the concept of an "energy-momentum" map, (see [15]), and obtaining a reduced system whose solutions give information on the bifurcation behaviour of his original system. We however, take a different approach than that followed by Van der Meer [15], by assuming that the Hamiltonian depends on a small parameter as in Section 2, obtaining the complex normal form and then doing a multiple scale analysis.

The case of the nonsemi-simple resonance is characterised by the fact that now the normal mode equations (2.4) possess a single normal mode solution at $\mu=0$.

$$
i \lambda \mathbf{a}_{1}=B_{0}^{T} \mathbf{a}_{1}+C_{0} \mathbf{b}_{1}, \quad i \lambda \mathbf{b}_{1}=B_{0} \mathbf{b}_{1}+A_{0} \mathbf{a}_{1}
$$

i.e. the system (2.4) has an additional degeneracy in that the "eigenvalue" $i \lambda$ has a single "eigenvector".

To analyse this case we proceed somewhat differently from section 2 in that we immediately derive the complex normal form and then proceed to an analysis of the fully nonlinear equations. To derive the complex normal form we first introduce the notion of a generalized normal mode vector $\left(\mathbf{a}_{2}, \mathbf{b}_{2}\right)$ defined according to

$$
i \lambda \mathbf{a}_{2}=B_{0}^{T} \mathbf{a}_{2}+C_{0} \mathbf{b}_{2}+\mathbf{a}_{1}, \quad-i \lambda \mathbf{b}_{2}=B_{0} \mathbf{b}_{2}+A_{0} \mathbf{a}_{2}-\mathbf{b}_{1} .
$$

From (3.1) and (3.2) we obtain the orthogonality conditions

$$
\mathbf{b}_{1}^{T} \overline{\mathbf{a}}_{1}-\mathbf{a}_{1}^{T} \overline{\mathbf{b}}_{1}=\overline{\mathbf{b}}_{1}^{T} \mathbf{a}_{2}-\mathbf{a}_{1}^{T} \mathbf{b}_{2}=0
$$

and

$$
\overline{\mathbf{b}}_{2}^{T} \mathbf{a}_{1}-\mathbf{a}_{2}^{T} \mathbf{b}_{1}=\alpha, \quad \overline{\mathbf{b}}_{2}^{T} \mathbf{a}_{2}-\overline{\mathbf{a}}_{2}^{T} \mathbf{b}_{2}=i \beta
$$

where $\alpha, \beta$ are real normalization constants which we are still free to choose.

To put the quadratic Hamiltonian into normal form we make the transformation

$$
\mathbf{q}=Q_{i} \mathbf{a}_{i}+\bar{Q}_{i} \overline{\mathbf{a}}_{i}, \quad \mathbf{p}=Q_{i} \mathbf{b}_{i}+\bar{Q}_{i} \overline{\mathbf{b}}_{i}
$$


and substitute these expressions into the $O(1)$ quadratic Hamiltonian

$$
H=(1 / 2) \mathbf{q}^{T}\left(A_{0} \mathbf{q}+B_{0} \mathbf{p}\right)+(1 / 2) \mathbf{p}^{T}\left(B_{0}^{T} \mathbf{q}+C_{0} \mathbf{p}\right) .
$$

Making use of the orthogonality conditions (3.3) and (3.4) we obtain

$$
H\left(Q_{i}, \bar{Q}_{i}\right)=i \alpha \lambda\left(\bar{Q}_{2} Q_{1}-Q_{2} \bar{Q}_{1}\right)-(\lambda \beta+\alpha)\left|Q_{2}\right|^{2} .
$$

However, the transformation from the cannonial variables $(\mathbf{q}, \mathbf{p})$ to be variables $\left(Q_{i}, \bar{Q}_{i}\right)$ is not necessarily a canonical transformation. To check that such a transformation can be made canonical we make use of the method outlined by Whittaker, [17]. In this case a transformation from the variables $(\mathbf{q}, \mathbf{p})$ to the variables $(\mathbf{Q}, \mathbf{P})$ is canonical provided that the expression

$$
\delta p_{j} d q_{j}-d p_{j} \delta q_{j}=\delta P_{j} d Q_{j}-d P_{j} \delta Q_{j}
$$

holds. Here $\left(\delta p_{j}, \delta q_{j}\right)$ and $\left(d p_{j}, d q_{j}\right)$ are independent infinitesimal variations in the $\left(p_{j}, q_{j}\right)$. In our case we have

$$
\delta p_{j} d q_{j}-d p_{j} \delta q_{j}=\alpha\left(\delta P_{j} d Q_{j}-d P_{j} \delta Q_{j}\right)+i \beta\left(\delta \bar{Q}_{2} d Q_{2}-\delta Q_{2} d \bar{Q}_{2}\right)
$$

where

$$
P_{1}=\bar{Q}_{2}, \quad P_{2}=-\bar{Q}_{1} .
$$

Thus, in order for the transformation from $(\mathbf{q}, \mathbf{p})$ to $(\mathbf{Q}, \mathbf{P})$ (with $\mathbf{P}$ given by (3.6)) to be canonical we must choose $\alpha$ and $\beta$ such that

$$
\alpha=1, \quad \beta=0 \text {. }
$$

In this case the $O(1)$ quadratic normal form is given by

$$
H\left(Q_{i}, \bar{Q}_{i}\right)=i \lambda\left(\bar{Q}_{2} Q_{1}-Q_{2} \bar{Q}_{2}\right)-\left|Q_{2}\right|^{2} \text {. }
$$

This normal form is equivalent (after some rescaling and a transformation to complex conjugate variables) to that presented by Arnold, [1] and Vad der Meer, [15].

To proceed with the analysis we write the full Hamiltonian as

$$
H=H_{2}^{(0)}+\mu H_{2}^{(1)}+H_{k}
$$

where $H_{2}^{(0)}$ is given by (3.7), $H_{2}^{(1)}$ is homogeneous of degree 2 and $H_{k}$ is homogeneous of degree $k$. Making use of the method of Lie transforms (see Appendix A) This Hamiltonian can be reduced to the normal form

$$
\begin{aligned}
H= & i\left(\lambda+\mu \nu_{1}\right)\left(\bar{Q}_{2} Q_{1}-Q_{2} \bar{Q}_{1}\right)-\left|Q_{2}\right|^{2}+\mu \nu_{2}\left|Q_{1}\right|^{2} \\
& +\alpha_{1}\left|Q_{1}\right|^{4}+i \alpha_{2}\left|Q_{1}\right|^{2}\left(Q_{1} \bar{Q}_{2}-\bar{Q}_{1} Q_{2}\right) \\
& +\alpha_{3}\left[3\left(Q_{1} \bar{Q}_{2}-Q_{2} \bar{Q}_{1}\right)^{2}+\left(Q_{1} \bar{Q}_{2}+\bar{Q}_{1} Q_{2}\right)^{2}\right]
\end{aligned}
$$


where $\nu_{1}$ and $\nu_{2}$ are parameters and the $\alpha_{i}$ are real constants. Note that $0<\mu \ll 1$ is a small parameter. The higher order corrections to the normal frequencies are then given explicitly by

$$
\omega_{1,2}=\left(\lambda+\mu \nu_{1}\right) \pm i \mu^{1 / 2} \nu_{2}^{1 / 2} .
$$

Thus, we see that the stability is governed by the sign of $\nu_{2}$; as $\nu_{2}$ changes sign the linearized normal freguencies go from stable to unstable. As noted by Van der Meer, [15], the Hamiltonian (3.8) is essentially an unfolding of the $O(1)$ quadratic Hamiltonian (3.7) and it this unfolding that is responsible for the loss of stability of the leading order normal mode.

Perturbation analysis. Hamilton's canonical equations for the Hamiltonian (3.8) are given by

$$
\begin{gathered}
d Q_{1} / d t=i\left(\lambda+\mu \nu_{1}\right) Q_{1}-Q_{2}+i \alpha_{2} Q_{1}\left|Q_{1}\right|^{2}+4 \alpha_{3} Q_{1}\left(2 Q_{1} \bar{Q}_{2}-Q_{2} \bar{Q}_{1}\right), \\
d Q_{2} / d t=i\left(\lambda+\mu \nu_{1}\right) Q_{2}-\mu \nu_{2} Q_{1}-2 \alpha_{1} Q_{2}\left|Q_{1}\right|^{2} \\
-\left(i \alpha_{2} Q_{1}-4 \alpha_{3} Q_{2}\right)\left(Q_{1} \bar{Q}_{2}-2 Q_{2} \bar{Q}_{1}\right) .
\end{gathered}
$$

We note that this system possesses the second integral of motion (the action integral)

$$
\bar{Q}_{2} Q_{1}-Q_{2} \bar{Q}_{1}=\text { constant }
$$

so that the system governed by the Hamiltonian (3.8) is integrable in the classical sense. Substituting

$$
Q_{j}=X_{j} \exp \left[i\left(\lambda+\mu \nu_{1}\right) t\right]
$$

into (3.9) and (3.10) yields the evolution equations

$$
d X_{1} / d t=-X_{2}+i \alpha_{2} X_{1}\left|X_{1}\right|^{2}+4 \alpha_{3} X_{1}\left(2 X_{1} \bar{X}_{2}-X_{2} \bar{X}_{1}\right)
$$

and

$$
d X_{2} / d t=-\mu \nu_{2} X_{1}-2 \alpha_{1} X_{1}\left|X_{1}\right|^{2}-\left(i \alpha_{2} X_{1}-4 \alpha_{3} X_{2}\right)\left(X_{1} \bar{X}_{2}-2 X_{2} \bar{X}_{1}\right) .
$$

This effectively averages out the oscillatory component of the solutions $Q_{i}$, $\bar{Q}_{i}$ of (3.9) and (3.10). Introducing the scaling

$$
\tau=\mu^{1 / 2} t, \quad X_{1}=\mu^{1 / 2} Y_{1}, \quad X_{2}=\mu Y_{2}
$$

and substitution into (3.11) and (3.12) yields to leading order in $\mu$

$$
d Y_{1} / d \tau=-Y_{2}, \quad d Y_{2} / d \tau=-\nu_{2} Y_{1}-2 \alpha_{1} Y_{1}\left|Y_{1}\right|^{2}
$$

or equivalently

$$
d^{2} Y_{1} / d \tau^{2}=\nu_{2} Y_{1}+2 \alpha_{1} Y_{1}\left|Y_{1}\right|^{2}
$$

with $\alpha_{1}$ and $\nu_{2}$ real. We note here that the only nonlinear coefficient $\alpha_{i}$ which appears in equation (3.14) is $\alpha_{1}$, the coefficient $\left|Q_{1}\right|^{4}$ in the Hamiltonian (3.8). 
This is in agreement with Van der Meer, [15], whose analysis shows that the dominant nonlinear coefficient is precisely this one.

Equation (3.14) arises in the analysis presented in Section 2(c) above and the properties of its solutions have been described there. We can show that these results are in agreement with those noted by Meyer, [13]. Equation (2.27) together with the restrictions on the critical points, $S_{0}$, correspond to Van der Meer's, [15], reduced system.

\section{Discussion}

We have show that the interaction through a resonance of two linear normal modes of a general Hamiltonian system as the system parameters are varied has the effect of producing an unstable mode in some region of the parameter space. The onset of this instability causes the linear normal mode approximation to become invalid. In this region of the parameter space one must now consider the fully nonlinear problem.

It is shown, using simple normal form theory and perturbation theory that the fully nonlinear problem can possess periodic solutions in the parameter range for which the linear normal modes are unstable. Agreement with earlier results has been demonstrated and both the semi-simple and nonsemi-simple cases were shown, locally about the point of resonance, to governed by the same form of second order differential equation.

Also, in the case of the semi-simple resonant interaction we have derived a compatibility condition on the nonlinear coefficients which determines whether it is possible for solutions to develop algebraic singularities at some finite time. This compatibility condition is independent of the parameter governing linear stability.

\section{Appendix A}

In this section we explicitly derive the higher order normal forms for both the semi-simple and nonsemi-simple case. We make use of the method of Lie transforms as outlined in [9], [11]. To this end we let $H_{2}^{(0)}$ denote the leading order quadratic Hamiltonian (i.e. $\mu=0$ ). We define the map $a d H_{2}^{(0)}$ according to

$$
\operatorname{ad~} H_{2}^{(0)}(F)=\left\{H_{2}^{(0)}, F\right\}
$$

where the Poisson bracket $\{\bullet, \bullet\}$ is defined according to

$$
\{G, F\}=\sum\left(\frac{\partial F}{\partial q_{i}} \frac{\partial G}{\partial p_{i}}-\frac{\partial F}{\partial p_{i}} \frac{\partial G}{\partial q_{i}}\right)
$$


The normal form is then found by obtaining a basis for the complement to the space $a d H_{2}^{(0)}\left(\mathbf{P}_{k}\right) ; \mathbf{P}_{k}$ is the space of polynomials of degree $k$. A suitable choice for this complement is the space $\operatorname{kerad~}_{2}^{(0)}\left(\mathbf{P}_{k}\right)$, the kernel of the map $\operatorname{ad} H_{2}^{(0)}\left(\mathbf{P}_{k}\right)$, (see $[9,11]$ for details).

Semi-simple case. The quadratic normal form for the semi-simple case is given by

$$
H_{2}^{(0)}=\lambda\left(\left|Q_{1}\right|^{2}-\left|Q_{2}\right|^{2}\right)
$$

where

$$
Q_{1}=p_{1}+i q_{1}, \quad Q_{2}=p_{2}+i q_{2} .
$$

In terms of the complex variable $Q_{i}, \bar{Q}_{i}$ the map ad $H_{2}^{(0)}$ becomes

$$
a d H_{2}^{(0)}=\frac{i}{2} \lambda\left(Q_{1} \frac{\partial}{\partial Q_{1}}-\bar{Q}_{1} \frac{\partial}{\partial \bar{Q}_{1}}-Q_{2} \frac{\partial}{\partial Q_{2}}+\bar{Q}_{2} \frac{\partial}{\partial \bar{Q}_{2}}\right) .
$$

The Hamiltonian can be reduced to its normal form by applying the map $a d H_{2}^{(0)}$ to the order $n$ monomials

$$
(k, l)=Q_{1}^{k_{1}} Q_{2}^{k_{2}} \bar{Q}_{1}^{l_{1}} \bar{Q}_{2}^{l_{2}}, \quad k_{1}+k_{2}+l_{1}+l_{2}=n .
$$

Allowing $a d H_{2}^{(0)}$ to act on these monomials we obtain

$$
\operatorname{ad} H_{2}^{(0)}(k, l)=-i \lambda\left(k_{1}=l_{1}-k_{2}-l_{2}\right)(k, l) .
$$

Hence

$$
\operatorname{kerad} H_{2}^{(0)}(k, l)=\left\{(k, l): k_{1}+l_{2}=l_{2}+k_{2}\right\}
$$

Thus

$$
\left.\operatorname{kerad} H_{2}^{(0)}\right|_{\mathbf{P}_{2}}=\left\{(k, l): k_{1}+l_{2}=l_{1}+k_{2}=1\right\}
$$

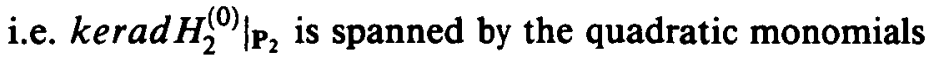

$$
Q_{1} Q_{2}, \bar{Q}_{1} \bar{Q}_{2},\left|Q_{1}\right|^{2},\left|Q_{2}\right|^{2} \text {. }
$$

Thus all $O(\mu)$ quadratic terms other than those given in (A.4) can be removed from the Hamiltonian by a canonical transformation. Also

$$
\text { kerad }\left.H_{2}^{(0)}\right|_{P_{3}}=\{0\} \text {. }
$$

Similarly

$$
\left.\operatorname{kerad} H_{2}^{(0)}\right|_{P_{4}}=\left\{(k, l): k_{1}+l_{2}=l_{1}+k_{2}=2\right\},
$$

and so the quartic Hamiltonian can be reduced, via a contact transformation, to a linear combination of the quartic monomials

$$
\begin{gathered}
\left|Q_{1}\right|^{4},\left|Q_{2}\right|^{4},\left|Q_{1}\right|^{2}\left|Q_{2}\right|^{2}, Q_{1}^{2} Q_{2}^{2}, \bar{Q}_{1}^{2} \bar{Q}_{2}^{2} \\
\left|Q_{2}\right|^{2} \bar{Q}_{1} \bar{Q}_{2},\left|Q_{1}\right|^{2} Q_{1} Q_{2},\left|Q_{1}\right|^{2} \bar{Q}_{1} \bar{Q}_{2},\left|Q_{2}\right|^{2} Q_{1} Q_{2}
\end{gathered}
$$


Nonsemi-simple case. The quadratic normal form for the nonsemi-simple case is given by

$$
H_{2}^{(0)}=i \lambda\left(\bar{Q}_{2} Q_{1}-Q_{2} \bar{Q}_{1}\right)-\left|Q_{2}\right|^{2}
$$

where

$$
Q_{1}=q_{1}+i q_{2}, \quad Q_{2}=p_{1}+i p_{2} .
$$

In terms of the complex variables $Q_{i}, \bar{Q}_{i}$ the map ad $H_{2}^{(0)}$ becomes

$$
\text { ad } H_{2}^{(0)}=2 i \lambda\left(Q_{j} \frac{\partial}{\partial Q_{j}}-\bar{Q}_{j} \frac{\partial}{\partial \bar{Q}_{j}}\right)-2\left(Q_{2} \frac{\partial}{\partial Q_{1}}+\bar{Q}_{2} \frac{\partial}{\partial \bar{Q}_{1}}\right)
$$

Quadratic normal form. The $O(\mu)$ quadratic Hamiltonian $H_{2}^{(1)}$ can be reduced to its normal form by applying the map ad $H_{2}^{(0)}$ to the quadratic monomials

$$
(k, l)=Q_{1}^{k_{1}} Q_{2}^{k_{2}} \bar{Q}_{1}^{l_{1}} \bar{Q}_{2}^{l_{2}}, \quad\left(k_{1}+k_{2}+l_{2}+l_{2}=2\right) .
$$

We compute the image of the map ad $H_{2}^{(0)}$ on the generators

$$
\begin{gathered}
X_{1}=\left|Q_{1}\right|^{2}, \quad X_{2}=\left|Q_{2}\right|^{2}, \quad X_{3}=\left(Q_{1} \bar{Q}_{2}-Q_{2} \bar{Q}_{1}\right), \\
X_{4}=\left(Q_{1} \bar{Q}_{2}+Q_{2} \bar{Q}_{1}\right), \quad X_{5}=Q_{1}^{2}, \quad X_{6}=\bar{Q}_{1}^{2}, \\
X_{7}=Q_{2}^{2}, \quad X_{8}=\bar{Q}_{2}^{2}, \quad X_{9}=Q_{1} Q_{2}, \quad X_{10}=\bar{Q}_{1} \bar{Q}_{2} .
\end{gathered}
$$

The matrix representing the action of $a d H_{2}^{(0)}$ on these generators is given by

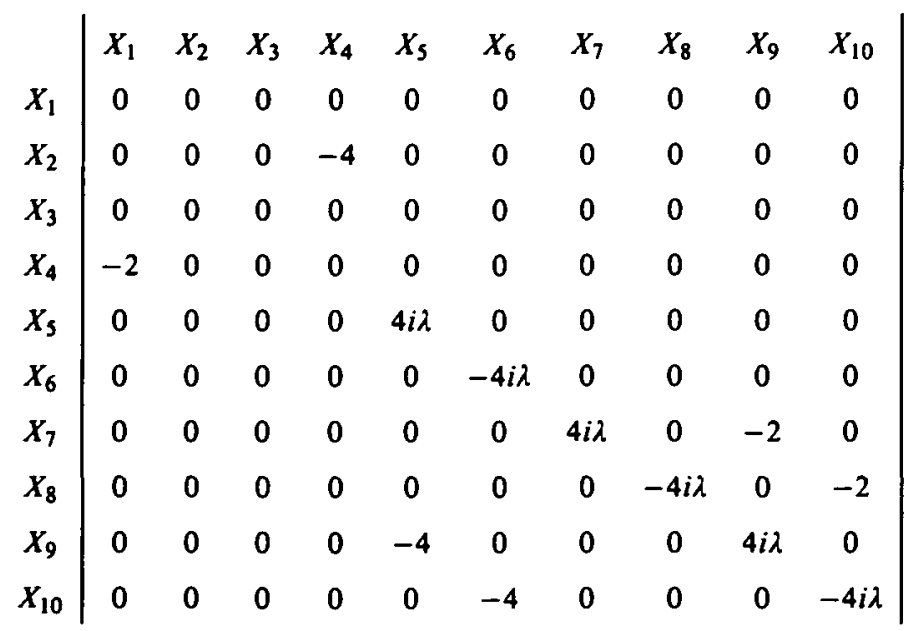


This matrix is singular. Columns 1, 4-10 are linearly independent and a complement for $a d H_{2}^{(0)}$ can be chosen as the space spanned by the monomials represented by the left eigenvectors of the above matrix

$$
(10000000000)^{T},(00100000000)^{T} \text {. }
$$

Thus a complement to the kernel of $a d H_{2}^{(0)}$ can be chosen to be spanned ùy tỉe mononoñinàis $X_{1}, X_{3}$. Hence, the $O(\mu)$ normai form for the quadratic Hamiltonian can be represented as a linear combination of the monomials $X_{1}$ and $X_{3}$ i.e.

$$
H_{2}^{(1)}=i \nu_{1}\left(\bar{Q}_{2} Q_{1}-Q_{2} \bar{Q}_{1}\right)+\nu_{2}\left|Q_{1}\right|^{2} .
$$

Without presenting the analysis for the cubic terms it is sufficient to note that all cubic terms can be removed from the Hamiltonian by a canonical change of coordinates.

Quartic normal form. To obtain the normal form of the fourth order Hamiltonian $\mathrm{H}_{4}$ it is sufficient to consider the action of the map ad $\mathrm{H}_{2}^{(0)}$ on the monomials

$$
\begin{array}{cc}
Z_{1}=\left|Q_{1}\right|^{4}, & Z_{2}=\left|Q_{2}\right|^{4}, \\
Z_{3}=\left|Q_{1}\right|^{2}\left(Q_{1} \bar{Q}_{2}+\bar{Q}_{1} Q_{2}\right), & Z_{4}=\left|Q_{2}\right|^{2}\left(Q_{1} \bar{Q}_{2}+\bar{Q}_{1} Q_{2}\right), \\
Z_{5}=\left|Q_{2}\right|^{2}\left(Q_{1} \bar{Q}_{2}-\bar{Q}_{1} Q_{2}\right), & Z_{6}=\left|Q_{1}\right|^{2}\left(Q_{1} \bar{Q}_{2}-\bar{Q}_{1} Q_{2}\right), \\
Z_{7}=\left(Q_{1} \bar{Q}_{2}-\bar{Q}_{1} Q_{2}\right)^{2}, & Z_{8}=\left(Q_{1} \bar{Q}_{2}+\bar{Q}_{1} Q_{2}\right)^{2}, \\
Z_{9}=\left(Q_{1} \bar{Q}_{2}-\bar{Q}_{1} Q_{2}\right)\left(Q_{1} \bar{Q}_{2}+\bar{Q}_{1} Q_{2}\right) .
\end{array}
$$

The matrix representing the action of $a d H_{2}^{(0)}$ on these monomials is

$$
\begin{array}{c|ccccccccc} 
& Z_{1} & Z_{2} & Z_{3} & Z_{4} & Z_{5} & Z_{6} & Z_{7} & Z_{8} & Z_{9} \\
Z_{1} & 0 & 0 & 0 & 0 & 0 & 0 & 0 & 0 & 0 \\
Z_{2} & 0 & 0 & 0 & -4 & 0 & 0 & 0 & 0 & 0 \\
Z_{3} & -4 & 0 & 0 & 0 & 0 & 0 & 0 & 0 & 0 \\
Z_{4} & 0 & 0 & 0 & 0 & 0 & 0 & 0 & -8 & 0 \\
Z_{5} & 0 & 0 & 0 & 0 & 0 & 0 & 0 & 0 & -4 \\
Z_{6} & 0 & 0 & 0 & 0 & 0 & 0 & 0 & 0 & 0 \\
Z_{7} & 0 & 0 & 1 & 0 & 0 & 0 & 0 & 0 & 0 \\
Z_{8} & 0 & 0 & -3 & 0 & 0 & 0 & 0 & 0 & 0 \\
Z_{9} & 0 & 0 & 0 & 0 & 0 & -1 & 0 & 0 & 0
\end{array}
$$


We observe that the vectors

$$
(100000000)^{T},(000001000)^{T},(000000310)^{T}
$$

are left eigenvectors for zero of the above matrix. Thus $a d H_{2}^{(0)}$ has a compliment which is at least three dimensional. Also columns $1,4,6,8,9$ are seen to be linearly independent. Thus the compliment of $a d H_{2}^{(0)}$ is three dimensional and is spanned by the three vectors (A.6). Thus the quartic normal form is given by

$$
\alpha_{1} Z_{1}+i \alpha_{2} Z_{6}+\alpha_{3}\left(3 Z_{7}+Z_{8}\right)
$$

\section{Acknowledgements}

During the course of this work J. P. Denier was the holder of a Commonwealth Postgraduate Research Award.

\section{References}

[1] V. I. Arnold, Mathematical methods in classical mechanics (Springer-Verlag, New York, 1978).

[2] R. A. Cairns, “The role of negative energy waves in some instabilities of parallel flows", $J$. Fluid Mech. 92 (1979) 1-14.

[3] A. A. Craik and J. A. Adams, “'Explosive' resonant wave interactions in a three-layer fluid", J. Fluid Mech. 92 (1979) 15-33.

[4] R. C. Davidson, Methods in nonlinear plasma theory, (Academic, New York, Vol. 37, Pure and Applied Physics, 1972)

[5] J. P. Denier, Ph. D. Thesis, University of New South Wales, 1989.

[6] R. H. J. Grimshaw, "Linearly coupled, slowly varying oscillators: the interaction of a positive energy mode with a negative energy mode", Studies in Appl. Math. 74 (1986) 205-226

[7] R. Grimshaw and J. S. Allen, "Linearly coupled, slowly varying oscillators", Studies in Appl. Math. 61, (1979) 55-71.

[8] R. Grimshaw, "Triad resonance for weakly coupled, slowly varying show oscillators", Studies in Appl. Math. 77 (1987) 1-35.

[9] J. Guckenheimer and P. Holmes, Nonlinear oscillations, dynamical systems and bifurcations of vector fields (Springer-Verlag, New York, 1983).

[10] G. looss and D. D. Joseph, Elementary stability and bifurcation theory (Springer-Verlag, New York, 1980).

[11] A. J. Lichtenberg and M. A. Lieberman, Regular and stochastic motion (Springer-Verlag, New York, 1983).

[12] A. A. Liapunov, "Problème général de la stabilitè du mouvement", in Annals of Math. Studies 17 (Princeton Univ. Press, 1947).

[13] K. R. Meyer, "Generic bifurcations in Hamiltonian systems", In Dynamical systems Warwick 1974 Lecture Notes in Mathematics, 468 (Springer-Verlag, New York, 1974). 
[14] J. W. Miles, "Weakly nonlinear Kelvin-Helmholtz waves", J. Fluid Mech. 172 (1986) 513529.

[15] J. C. Van der Meer, "The Hamiltonian Hopf Bifurcation", Lecture Notes in Mathematics, 1160 (Springer-Verlag, New York, 1986).

[16] __ "Nonsemisimple 1:1 resonance at an equilibrium", Cl. Mech. 27 (1982) 131-149.

[17] E. T. Whittaker, $A$ treatise on the analytic dynamics of particles and rigid bodies, 4th ed. (C. U. P, Cambridge, 1937). 